\title{
Fluorogenic peptides for the detection of programmed cell death
}

\author{
Ramon Subiros-Funosas ${ }^{1}$, Nicole Barth ${ }^{1}$, Jesus Sot ${ }^{2}$, Felix Goni ${ }^{2}$, Lorena Mendive-Tapia ${ }^{1}$, Rodolfo Lavilla ${ }^{3}$, Rodger \\ Duffin $^{1}$, Adriano Rossi ${ }^{1}$, Ian Dransfield ${ }^{1}$, Marc Vendrell ${ }^{1}$ \\ ${ }^{1}$ The University of Edinburgh, United Kingdom \\ ${ }^{2}$ Instituto Biofisika, Universidad del Pas Vasco, Spain \\ ${ }^{3}$ The University of Barcelona, Spain
}

https://doi.org/10.17952/35EPS.2018.056

Programmed cell death is an essential process in the development and progression of numerous diseases [1]. Therefore, there is a need for sensitive methods for the detection of apoptotic cells as mechanistic tools to unravel disease pathogenesis. The current gold standard for imaging apoptosis are fluorescently-labelled annexins, which display strong binding to phosphatidylserine (PS), a membrane phospholipid that is translocated to the external leaflet of the plasma membrane during the early stages of the apoptotic process [2,3]. However, annexins require high concentrations of $\mathrm{Ca}^{2+}$ for binding, which are not found in many physiological environments, including injured tissues [4]. Herein we developed peptide-based fluorogenic probes for $\mathrm{Ca}^{2+}$-independent imaging of cells undergoing programmed death.

We used amphipathic peptides as templates to generate optical probes binding PS in apoptotic cells while showing marginal binding to viable cells. Notably, fluorogenic staining was achieved by incorporation of the environmentally sensitive Trp-BODIPY amino acid, which fluoresces only in close proximity to the binding target [5]. We have recently reported this amino acid as an optimal fluorophore to label peptides with minimal disruption of their binding properties and capabilities for wash-free imaging in real time [6]. Moreover, TrpBODIPY displays excellent photophysical properties including emission in the green region of the visible spectra, matching GFP filters found in conventional microscopes.

We designed different fluorogenic peptides to study the hydrophilic and aromatic sequence requirements that defined optimal binding to PS. All peptides contained the Trp-BODIPY fluorogen while the nature of the amphipathic residues varied and included a broad range of polar and hydrophobic amino acids. We screened the peptides using lipid films in vitro, where the most promising binders displayed remarkable fluorescence emission in PS when compared to uncharged lipids or buffer. From a synthetic point of view, Trp-BODIPY is compatible with standard Fmoc/tBu SPPS protocols and can be incorporated using conventional coupling methods. However, the intrinsic lability of the BODIPY core under acid media require the use of acid labile solid supports (e.g. 2-chlorotritylchloride polystyrene resin) and side-chain protecting groups that can be removed in neutral conditions, such as hydrogenolysis.

All peptides were screened in cell mixtures containing $~ 50 \%$ apoptotic neutrophils and $\sim 50 \%$ viable neutrophils, and we compared their signal to background fluorescence in viable cells. F luorescence $f$ old increase (as a measure of selectivity for apoptotic over viable cells) and fluorescence after washing (as a measure of binding strength) were measured for all peptides. From these studies, we concluded that peptides containing positivelycharged residues bound strongly to apoptotic cells with fast kinetics, whereas negatively-charged ones did not show any binding to apoptotic or viable cells. We also observed that a balanced ratio of positive charges and hydrophobic residues was necessary to achieve rapid and selective binding to apoptotic cells. Some peptides exhibited strong binding to apoptotic neutrophils and low fluorescence emission in viable cells.

We further validated the peptides for detection of apoptotic cells from multiple origins and species. Furthermore, in contrast to Annexin V, these peptides stained apoptotic cells independently of $\mathrm{Ca}^{2+}$ concentration, with optimal performance even in the presence of the $\mathrm{Ca}^{2+}$ chelator EDTA. We further characterized cell populations based on their fluorescence staining, and confirmed that peptide-positive cells displayed features that are characteristic of apoptosis [7,8]. Finally, we used the peptides to detect apoptosis in mouse models. Peptide-stained cells were quantified by fluorescence confocal microscopy in tissue sections, revealing significant increase of apoptosis in inflamed tissues compared to healthy tissues. Notably, the peptides did not show any cytotoxic effect and did not impair clearance of dead cells by professional phagocytes, such as macrophages. In summary, we developed fluorogenic peptides for the detection of apoptosis under a broad spectrum of physiological environments and avoiding the need for high levels of extracellular $\mathrm{Ca}^{2+}$ that might facilitate their potential use for in vivo applications. 
References

[1] Poon, I.K., Lucas, C.D., Rossi, A.G. \& Ravichandran, K.S. Apoptotic cell clearance: basic biology and therapeutic potential. Nat Rev Immunol., 2014, 14, 166-180.

[2] Koopman, G., et al., Annexin V for flow cytometric detection of phosphatidylserine expression on B cells undergoing apoptosis. Blood, 1994, 84, 1415-1420.

[3] Subiros-Funosas, R., et al., A Trp-BODIPY cyclic peptide for fluorescence labelling of apoptotic bodies. Chem. Commun., 2017. 53(5), 945-948.

[4] Baird, G.S. Ionized calcium. Clin Chim Acta, 2011, 412, 696-701.

[5] Mendive-Tapia, L., et al., Spacer-free BODIPY fluorogens in antimicrobial peptides for direct imaging of fungal infection in humantissue. Nat. Commun., 2016. 7, 10940.

[6] Mendive-Tapia, L., et al., Preparation of a Trp-BODIPY fluorogenic amino acid to label peptides for enhanced live-cell fluorescence imaging. Nat. Protocols, 2017. 12(8),1588-1619.

[7] Knepper-Nicolai, B., Savill, J. \& Brown, S.B. Constitutive apoptosis in human neutrophils requires synergy between calpains and the proteasome downstream of caspases. J Biol Chem, 1998, 273, 30530-30536.

[8] Morris, R.G., Hargreaves, A.D., Duvall, E. \& Wyllie, A.H. Hormone-inducedcell death. 2. Surface changes in thymocytes undergoing apoptosis, Am J Pathol, 1984, 115, 426-436. 